\title{
Control of Shape and Size of Nanopillar Assembly by Adhesion-Mediated Elastocapillary Interaction
}

\section{Citation}

Kang, Sung H., Boaz Pokroy, L. Mahadevan, and Joanna Aizenberg. 2010. “Control of Shape and Size of Nanopillar Assembly by Adhesion-Mediated Elastocapillary Interaction." ACS Nano 4, no. 11: 6323-6331. doi:10.1021/nn102260t.

\section{Published Version}

doi:10.1021/nn102260t

\section{Permanent link}

http://nrs.harvard.edu/urn-3:HUL.InstRepos:33187427

\section{Terms of Use}

This article was downloaded from Harvard University's DASH repository, and is made available under the terms and conditions applicable to Open Access Policy Articles, as set forth at http:// nrs.harvard.edu/urn-3:HUL.InstRepos:dash.current.terms-of-use\#OAP

\section{Share Your Story}

The Harvard community has made this article openly available.

Please share how this access benefits you. Submit a story.

\section{Accessibility}




\title{
Control of Shape and Size of Nanopillar Assembly
}

\section{by Adhesion-mediated Elastocapillary Interaction}

\author{
Sung H. Kang, Boaz Pokroy, L. Mahadevan, and Joanna Aizenberg*
}

School of Engineering and Applied Sciences, Harvard University, 29 Oxford Street, Cambridge, Massachusetts 02138

E-mail: jaiz@seas.harvard.edu

\section{RECEIVED DATE}

*Corresponding author - Tel: (617) 495-3558, Fax: (617) 496-1457, E-mail: jaiz@seas.harvard.edu

ABSTRACT Control of self-organization of nanofibers into regular clusters upon evaporationinduced assembly is receiving increasing attention due to the potential importance of this process in a range of applications including particle trapping, adhesives, and structural color. Here we present a comprehensive study of this phenomenon, using a periodic array of polymeric nanopillars with tunable parameters as a model system to study how geometry, mechanical properties as well as surface properties influence capillary-induced self-organization. In particular, we show that varying the parameters of the building blocks of self-assembly provides us with a simple means of controlling the size, chirality and anisotropy of complex structures. We observe that chiral assemblies can be generated within a narrow window for each parameter even in the absence of chiral building blocks or a chiral environment. Furthermore, introducing 
anisotropy in the building blocks provides a way to control both the chirality and the size of the assembly. While capillary-induced self-assembly has been studied and modeled as a quasi-static process involving the competition between only capillary and elastic forces, our results unequivocally show that both adhesion as well as kinetics are equally important in determining the final assembly. Our findings provide insight into how multiple parameters work together in capillary-induced self-assembly and provide us with a diverse set of options for fabricating a variety of nanostructures by self-assembly.

KEYWORDS Self-Assembly, Hierarchical Structures, Nanorods, Polymeric Materials, Biomimetics

\section{Introduction}

Self-assembly is becoming increasingly important as a bottom-up route for building novel structures and controlling functions at length scales ranging from the nanometer to millimeter. ${ }^{1-3}$ As current miniaturization methods for microelectronics and robotics approach their theoretical limit and/or commercially feasible minimum size, self-assembly provides new fabrication and manufacturing strategies that can overcome these challenges. ${ }^{3}$ Consequently, much work has focused on the control of assembly at molecular scales, ${ }^{1-2,4}$ and there is currently a growing interest in understanding and manipulating the assembly process at larger (submicron to millimeter) length scales. Various driving forces for self-assembly have been studied at these larger length scales, ${ }^{5}$ including gravity, ${ }^{6}$ magnetic force, ${ }^{7}$ electrostatic interaction, ${ }^{8}$ entropy, ${ }^{9}$ and capillary interaction. ${ }^{10}$ Among these, capillary effects have received particular attention at the micro-scale because of their relatively significant magnitude, tunability and simplicity, ${ }^{5}$ and a number of studies have demonstrated the formation of complex two- and three-dimensional 
structures by this method. ${ }^{11-15}$ Among many building blocks, synthetic fibers have been selfassembled by capillarity in a wide range of systems, such as macroscopic hairs of a brush, ${ }^{16}$ a micron-scale polymeric surface mimicking the foot of the gecko ${ }^{17}$ as well as nanowires ${ }^{18}$ and nanotubes. ${ }^{19-20}$ However, there was little or no control of the assembly size or pattern in any of these artificial systems unless laborious e-beam pre-patterning of the array was used. ${ }^{21}$ Our study is inspired by a wide range of biological systems in which assembly of nanofibers has functional significance. Just as a tantalizing example, the beetle Hemisphaerota cyanea controls the assembly size of its tarsi (segmented feet) by adjusting the secretion of an oily liquid to adjust its adhesion to a surface..$^{22}$

We are interested in investigating the relation between the intrinsic features of the nanoscale building blocks and the final assembly pattern as a basis for selecting parameters that specify desired structures. Despite several works reporting the self-assembly of synthetic fibrous surfaces, relatively little is known about how the many parameters of the system influence the size and kinetics of self-assembly. In previous studies, the contributions of the properties of building blocks to the assembly process were described by competition between elasticity and capillarity for wet macroscopic systems ${ }^{16,23-24}$, as well as micropillars ${ }^{25-26}$ and nanoscopic systems such as carbon nanotubes ${ }^{19}$ and silicon nanorods. ${ }^{27}$ Two studies have analyzed the correlation between assembly size and the aspect ratio of the filaments, as well as their diameter and Young's modulus, and the surface tension of the liquid. ${ }^{25,27}$ In scaling terms, both studies used static energy minimization and derived the relationship:

$$
N \sim \frac{E_{C}}{E_{E}} \sim \frac{D^{2} \gamma \cos ^{2} \theta}{\frac{D^{4}(p-D)^{2} E}{h^{3}}} \sim \frac{\gamma h^{3} \cos ^{2} \theta}{D^{2}(p-D)^{2} E}
$$


where $N=$ average number of rods in one assembly, $E_{C}=$ capillary interaction energy, $E_{E}=$ elastic energy term, $h=$ height of the rods, $\gamma=$ surface tension of the liquid, $D=$ diameter of the rod, $p=$ distance between the rods, $E=$ Young's modulus of the rod, $\theta=$ equilibrium contact angle of the liquid on the surface of the rods. However, all these studies assumed that the assembled bundles stay together once they are formed and did not consider the effects of the adhesion even though it alone maintains the assembly after the system dries and capillary forces disappear, and also neglected any kinetic effects.

Going beyond the size of the assembled clusters, their shape may have important implications for nanofabrication of advanced materials. For example, hierarchical chiral architectures provide useful mechanical robustness in cellulose fibrils in $\operatorname{wood}^{28}$ and mineralized collagen fibrils in bone, ${ }^{29}$ or structural color, via complex polarization-sensitive structures in beetles ${ }^{30}$ and butterflies. ${ }^{31}$ Recently, we have reported the hierarchical chiral assembly of polymer bristles by evaporative self-assembly achieved without using chiral building blocks or a chiral environment and developed a model for the assembly process based on interaction of elastic, capillary and adhesion forces. ${ }^{32}$ Our theoretical analysis shows that adhesion between the posts by short range van der Waals forces is important in inducing chirality and determining the size and shape of the final clusters. Since this evaporation-induced chiral assembly of fibers has not been observed in other studies, it is critical to analyze further the factors and conditions that would lead to controlled formation of functionally important structures. Here, using periodic arrays of nanopillars with controlled modulus, geometry, and surface properties (Figure 1), we show how varying the parameters of the pillars associated with capillarity, elasticity and adhesion gives us simple ways of controlling the chirality, size, and shape of the assemblies. 
(a)
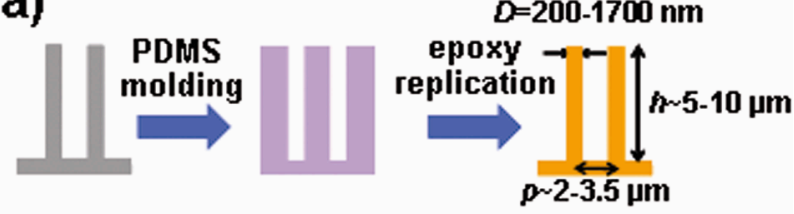

(b)
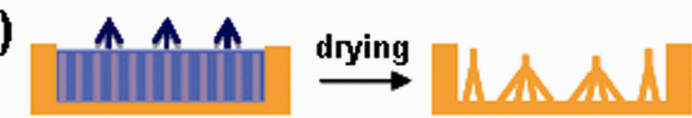

(c)

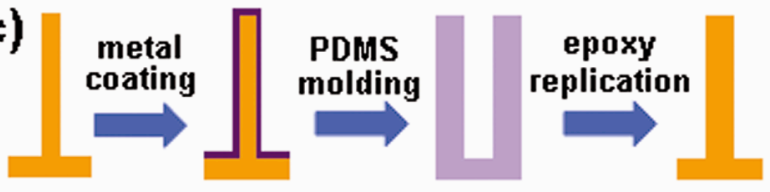

(d)

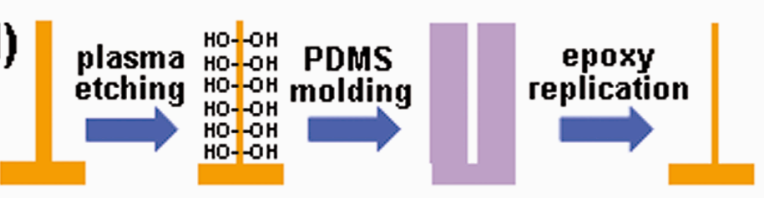

(e)

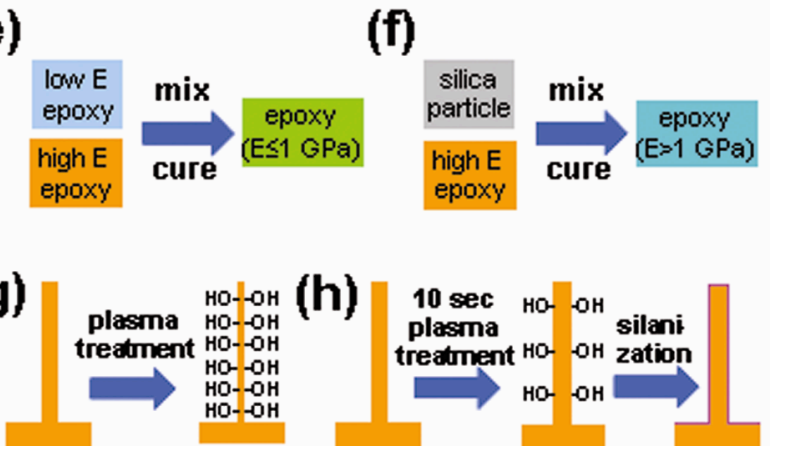

Figure 1. Schematics of the experimental procedures. (a) Schematic of the sample fabrication procedure and the geometry of the arrays of polymeric nanopillars used in the study. (b) Schematic of the assembly of nanopillars by evaporation of the applied liquid. (c)-(h) Schematics of the methods used to change the parameters of the posts (See Experimental Methods for details.): (c) Increasing the diameter, (d) Decreasing the diameter, (e) Decreasing the modulus of the post (E $\leq 1 \mathrm{GPa})$, (f) Increasing the modulus of the post $(\mathrm{E}>1 \mathrm{GPa}),(\mathrm{g})$ Increasing adhesion, (h) Changing the wetting property.

\section{Results}

Nanopillars were fabricated by producing negative elastomeric molds of the original fibrous arrays and replicating them in a polymer using the method described earlier (Figure $1 \mathrm{a}) .^{33}$ The polymeric nanopillars were then allowed to assemble as the liquid in which they were immersed evaporated (Figure 1b). To study the effects of elasticity of the posts, we varied the geometry (Figure 1c,d) or the modulus (Figure 1e,f). The effect of adhesion was assessed either by using 
plasma treatment to activate reactive surface functional groups (Figure 1g) or by chemical functionalization of the surface (Figure 1h).

Pillar size. To systematically increase the diameter of the nanofibers, the pillars were coated with a metal film of a controlled thickness. This changes both the surface properties and modulus of the resulting pillars, while increasing the diameter. To decrease the diameter of the pillars, the surfaces were etched in oxygen plasma for controlled periods of time. This reduces the pillar diameter and also changes the surface properties and therefore adhesive and capillary interaction in the assembling system. To decouple the effect of the geometry from the effect of adhesion, surface tension and stiffness, both metal-coated and plasma-etched structures were further replicated in the original polymer (Figure 1c,d). When only the diameter of a post was increased, while other parameters were kept constant (modulus: $E \sim 1 \mathrm{GPa}$, height: $h \sim 8 \mu \mathrm{m}$, and pitch: $p \sim 2 \mu \mathrm{m}$ ), the number of posts per assembly decreased (Figure 2a-d) so that $N^{-1} \sim D^{2}(p$ $D)^{2}$, as shown in Figure $2 \mathrm{~d}$ consistent with Eq. (1), since changing the diameter also alters the spacing between the posts and, as a result, both the capillary force and the elastic force ${ }^{25,}{ }^{27}$ For large pillar diameters, the final size of the assembly in the dry sample falls slightly below the expected value. Careful analysis of the scanning electron microscope (SEM) images (Figure 2c) shows that the assemblies have some anisotropy so that although larger assemblies were formed initially, they were subsequently divided into smaller clusters. Optical microscope studies confirmed that a bigger assembly was indeed formed when the system was wet, but divided into sub-clusters when the system dried. This result suggests that the surface adhesion is not strong enough to accommodate the increase in pillar elasticity. The shape of the assembled clusters, however, does not show any gradual transformation with pillar diameter: chirality appears only for a narrow range of intermediate diameters. 


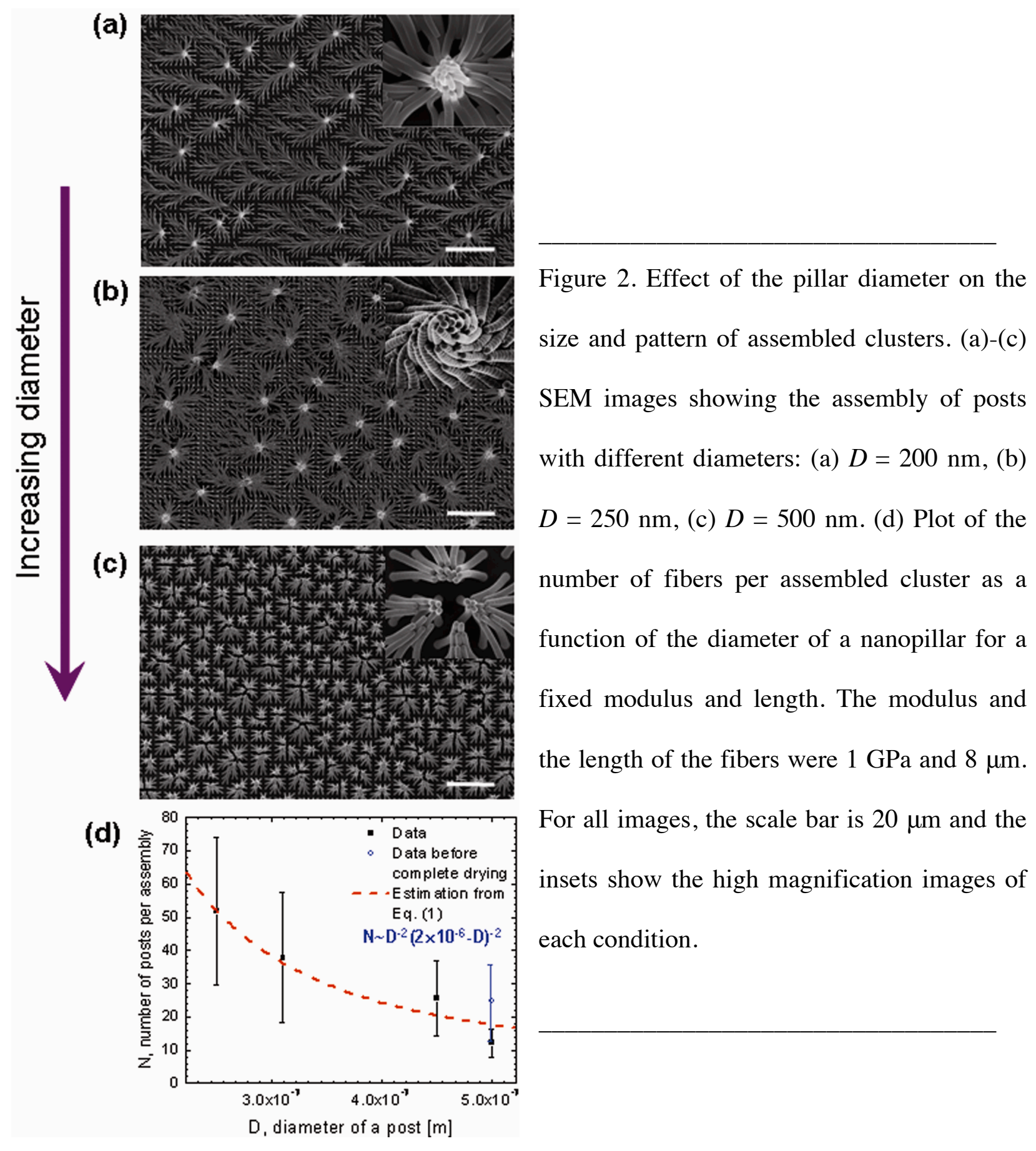

Anisotropic pillar arrays. We now extend our study to include the role of anisotropy in pillar cross-section and spacing as commonly seen in biologically occurring fibrous surfaces used for 
sensing,${ }^{34}$ adhesion, ${ }^{35}$ and superhydrophobicity ${ }^{36}$ The simplest way to include the anisotropy is to fabricate pillar arrays by deforming the elastomeric mold. ${ }^{33}$ Applying force along a lattice direction to the original mold with the circular holes as illustrated in Figure 3a causes the holes to become elliptical and change the lattice parameters. This leads to pillars with an elliptical cross-section that assemble into anisotropic clusters as in Figure 3a. Analyzing the SEM images for the size of the assembly along the short and the long lattice axes allows us to decouple the effects of lattice anisotropy and the flexural stiffness anisotropy and shows that the ratio of the cluster sizes along both axes $\left(N_{1} / N_{2}\right)$ scales with the square of the ratio of the aspect ratio of the cross-section times the square of the ratio of the spacings between posts as below (See supporting information for details.), consistent with Eq. (1).

$$
\frac{N_{1}}{N_{2}} \sim 3.1 \pm 1.5 \approx\left(\frac{E_{C}}{E_{E}}\right)_{1} /\left(\frac{E_{C}}{E_{E}}\right)_{2}=\left(\frac{D_{2}}{D_{1}}\right)^{2}\left(\frac{p_{2}-D_{2}}{p_{1}-D_{1}}\right)^{2} \sim(1.6 \pm 0.8)^{2}(1.1)^{2}
$$

In addition to changing the cross-section and the spacing of the post arrays, we can also introduce anisotropy in the array by using tilted posts. ${ }^{33}$ Such an approach allows for the fine tuning of the size and shape of the assembled clusters. For example, a square array of nanopillars (modulus: $E \sim 1 \mathrm{GPa}$, height: $h \sim 5 \mu \mathrm{m}$, and pitch: $p \sim 2 \mu \mathrm{m}$ ) that forms tetramers similar to Figure $3 \mathrm{~b}$ if there is no tilting, will assemble into achiral dimers by using posts tilted along a lattice direction (Figure 3c). This form of anisotropy can also be utilized to control the chirality. by tilting the posts slightly off the lattice direction, ${ }^{32}$ leading to dimers with uniform chirality as shown in Figure 3d. 

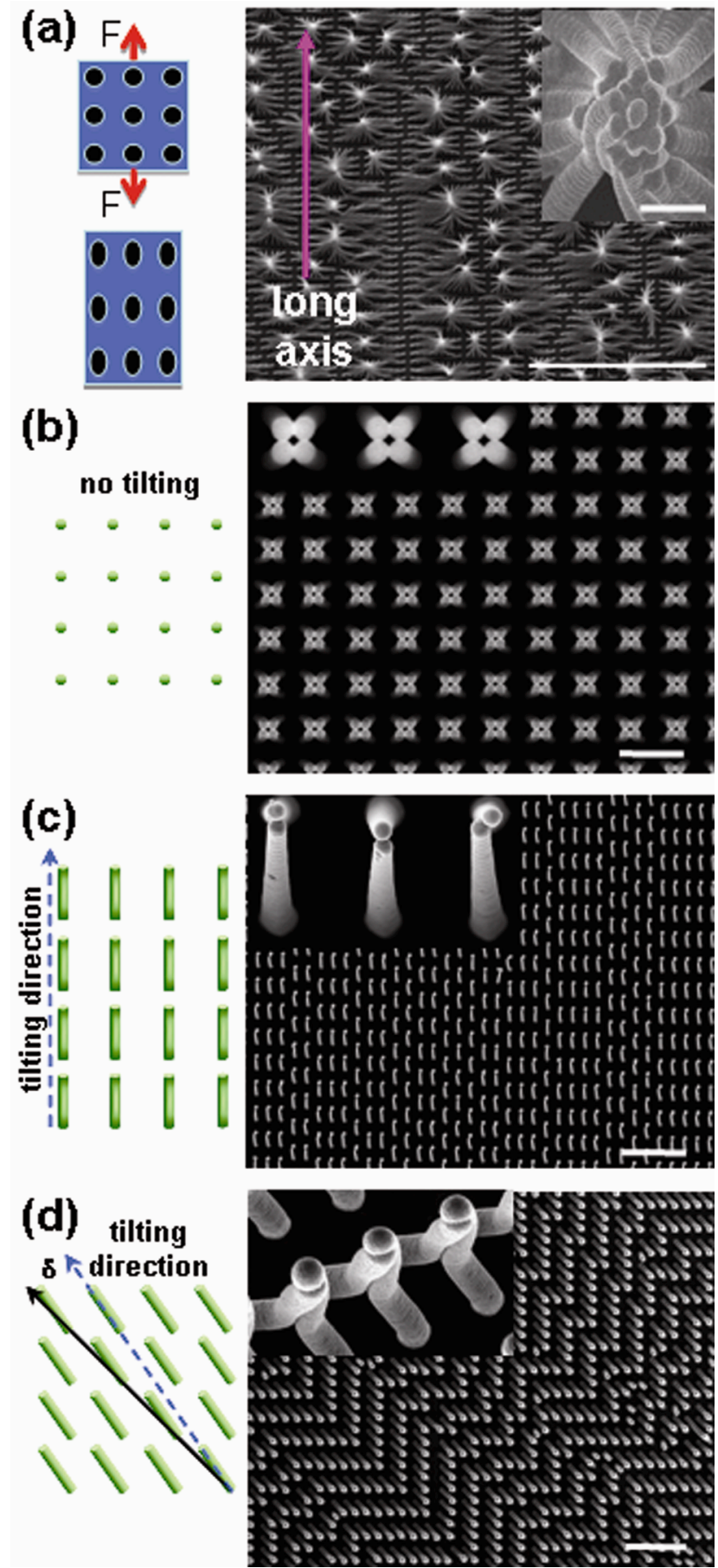

form chiral dimers with

Figure 3. Effect of the anisotropy on the size and pattern of assembled clusters. Left column shows schematics (not drawn to scale) of the method used to fabricate various nanopillar arrays and right column shows SEM images of the assemblies of corresponding nanopillar arrays. The insets show the highmagnification image of the corresponding SEM image. (a) Elliptical cross-section nanopillars $(h=8 \mu \mathrm{m})$ arranged into a rectangular lattice assemble into anisotropic clusters elongated in the direction of the short axis if the ellipse. (Scale bar: $50 \mu \mathrm{m}$ ). The long axis indicates the direction in which the tensile force was applied. The inset shows the elliptical cross-section of the posts. (Scale bar: $1 \mu \mathrm{m})$ (b) A square array of cylindrical nanopillars oriented perpendicular to the surface assemble into a regular array of tetramers. (c) The pillars tilted along a lattice direction form a regular array of achiral dimers instead of tetramers. (d) The pillars tilted slightly off a diagonal lattice direction a uniform twisting direction. Scale bars: $10 \mu \mathrm{m}$.

Elastic modulus of pillars. In addition to changing the diameter of the pillars or symmetry of 
the array, we studied the effect of the elasticity by varying the elastic modulus of the material

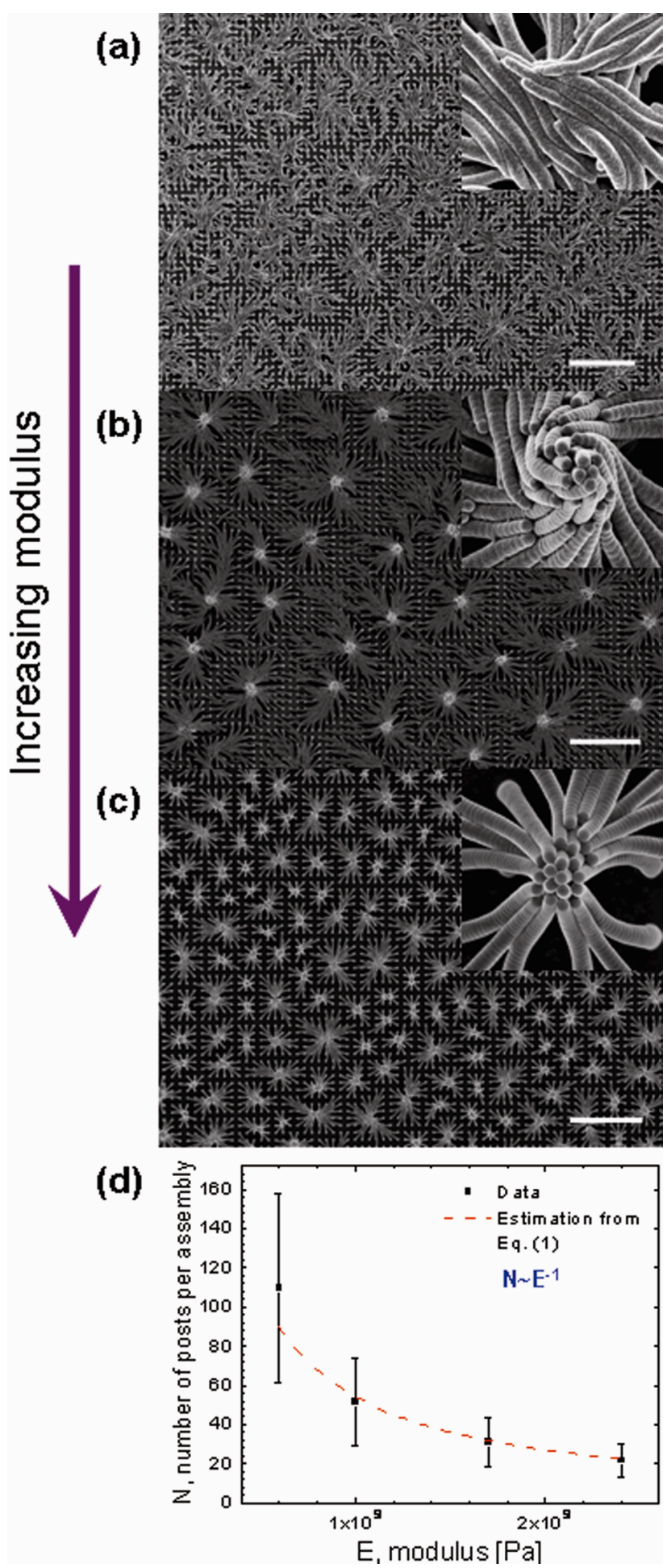

(Figure 1e,f). To systematically change the modulus of the pillars, two approaches were used: (i) adding the softener to the original polymer to decrease the stiffness of the pillars (Figure 1e), and (ii) mixing the polymer with silica nanoparticles to increase the stiffness of the pillars (Figure 1f). Note that while the former approach may also change the adhesive properties of the system, the latter would primarily affect the elastic modulus.

Figure 4. Effect of the pillar modulus on the size and pattern of assembled clusters. (a)-(c) Scanning electron microscope (SEM) images of assembly of fiber array with different moduli (E): (a) E 400MPa, (b) E 1GPa, (c) E $2.4 \mathrm{GPa}$. The diameter and the length of the fibers were fixed to $250 \mathrm{~nm}$ and $8 \mu \mathrm{m}$, respectively. The scale bars are $20 \mu \mathrm{m}$. The insets show high magnification images of the assemblies, which were used to check the onset of the chirality for each condition. (d) The plot of the number of posts per assembly as a function of the modulus for fixed diameter and length of the post. The fitting shows $1 / \mathrm{E}$ dependence. 
Changing the modulus showed a trend similar to changing the diameter; that is, appearance of chirality at intermediate modulus values, disordered achiral structures at a low modulus, and ordered achiral structures at a high modulus. The size of the assembly decreased with increasing modulus as shown in the representative SEM images (Figure 4a-c) and in the statistical analysis (Figure 4d) for the moduli tested (E $400 \mathrm{MPa}, 600 \mathrm{MPa}, 1 \mathrm{GPa}, 1.6 \mathrm{GPa}$, and $2.4 \mathrm{GPa}$ ). From the analysis, the number of posts per assembly is inversely proportional to the modulus. In addition, when either modulus or diameter increases, the observed range of the assembly sizes decreases, consistent with a previous study on aggregation of bundles of wet hair, showing that the range is proportional to the maximum assembly size. ${ }^{37}$

Effect of adhesion and wetting. Compared to the effects of elastic and capillary force terms, the effect of changing the adhesion force term has received little attention. We varied the adhesion of the pillars by using plasma treatment of the polymeric samples to activate reactive surface functional groups (Figure $1 \mathrm{~g}$ ) ${ }^{38}$ Though we do not have quantitative values for the resulting adhesion between the posts (currently under investigation), we expect that adhesion increases with plasma treatment time, as assessed by a qualitative comparison of forces required to slide two fibrous surfaces facing each other after making contacts. We observed that as the plasma treatment time increased, the chirality disappeared in the samples that showed substantial twisting when untreated. The assembled pillars then zipped parallelly instead of twisting helically, and the size of the clusters decreased as shown in Figure 5. This decrease in the size of 
the assemblies is somewhat counterintuitive, since in addition to increasing adhesion, plasma treatment decreases the diameter of the pillars and is therefore expected to increase the cluster size, if purely elasto-capillarity arguments (Eq. 1) are used (Figure 5d). However, if plasma treatment increases the adhesion sufficiently, this more than makes up for the decrease in the diameter.

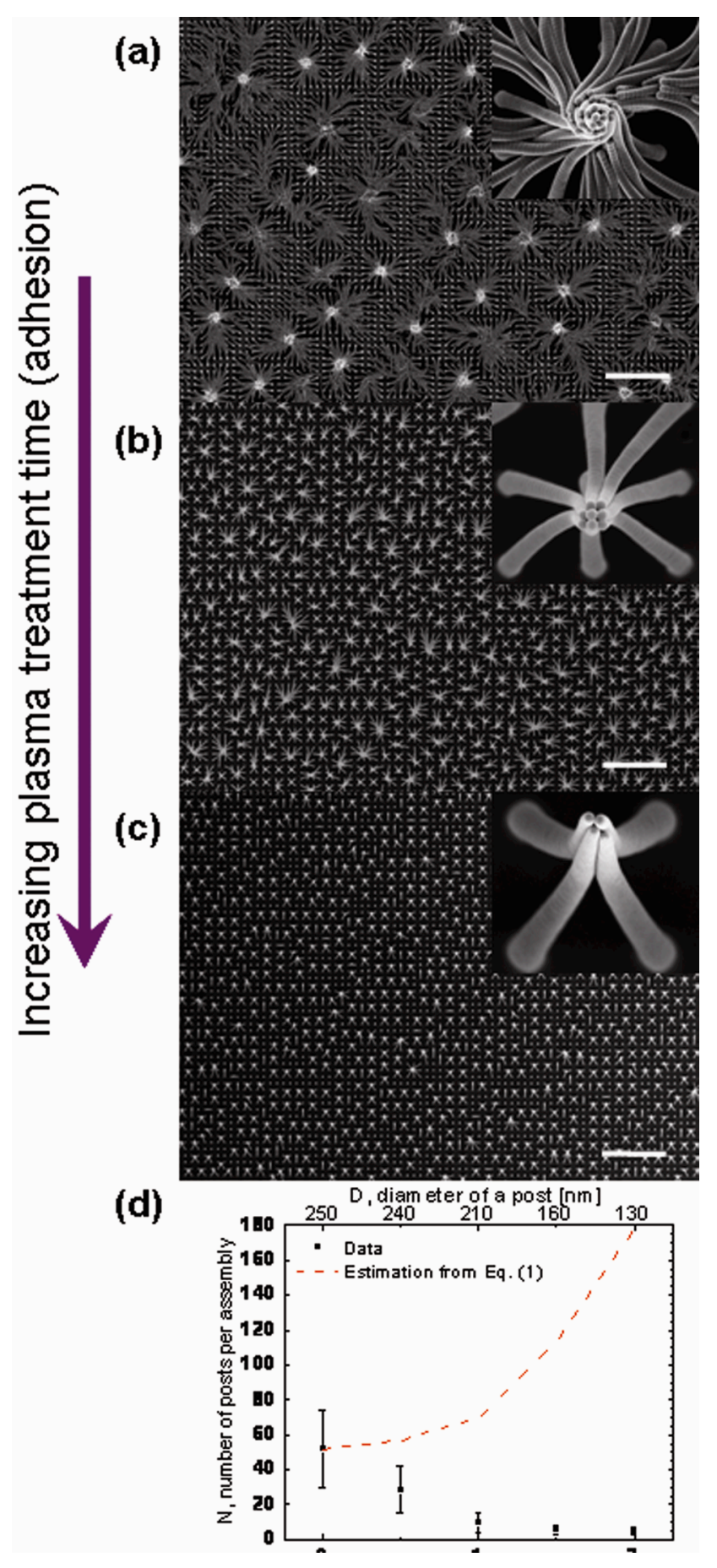

Figure 5. Effect of the plasma treatment on the size and pattern of assembled clusters. (a)-(c) SEM images of assembly of post arrays with different plasma treatment time. The plasma treatment increases adhesion as well as decreases the diameter of the nanopillars. (a) No plasma treatment (control). (b) $1 \mathrm{~min}$ plasma treatment. (c) 2 min plasma treatment. The modulus and the height of the pillars were fixed at $1 \mathrm{GPa}$ and $8 \mu \mathrm{m}$, respectively. The scale bars are $20 \mu \mathrm{m}$. The insets show the high magnification images used for determining the shape of individual clusters. (d) The plot of the number of posts per assembly as a function of plasma treatment time. Because the plasma treatment changes the adhesion and the diameter of the pillars at the same time, the corresponding diameters are shown on the top axis of the graph for a given plasma treatment time. Note that the number of posts per assembly decreases as the plasma treatment time increases even though the diameter of the pillars decreases. 
It is possible that the plasma treatment may also change the interfacial tension by increasing surface wetting. However, since the contact angle of ethanol on the as-fabricated nanopost array is already lower than $10^{\circ}$, its effect on the interfacial tension is negligible. In contrast, the effects of silanes of different critical surface tension values (Figure 1h) (see experimental and supporting information for details) does change the wetting properties substantially; as the surface tension decreases below the critical value, the chirality of the assembly disappears, and its size is significantly reduced (Figure S1).

Finally, increasing the adhesion by using a smoother surface, which has a larger contact area (Figure S2) causes structures to assemble like a zipper with no chirality. Thus we see that interpillar adhesion has the ability to control the chirality, shape and size of the final assembly after contacts are made.

\section{Discussion}

While evaporation-induced self-assembly has been studied and modeled as the result of the competition between capillary and elastic forces, ${ }^{17,}{ }^{23-26}$ our data demonstrate that inter-pillar adhesion is equally important to determining the final assembly. Our instantaneous and continuous real-time imaging show that assembly is, in fact, biphasic: posts assemble hierarchically, as previously reported, ${ }^{32}$ but can subsequently undergo hierarchical disassembly as outlined in Figure 6a. We observe this second phase most distinctly when the pillar stiffness is relatively high (Figure 2c); a large assembly initially forms under capillary force, but, after the 
liquid dries, it breaks apart into smaller clusters equal to its immediate precursors. Since adhesion alone balances the elastic deformation in the dry system, the most straightforward conclusion is that adhesion is insufficient to maintain the last stage of assembly. Thus, while the balance between capillarity and elasticity determines the maximum size the system can achieve,,$^{25-27,37,39}$ the balance between adhesion and elasticity determines how many hierarchical stages will be preserved in the final structure. ${ }^{32}$ The fact that disassembly follows a reverse hierarchical path, rather than one-step dissociation, suggests that the assembly size is specified by the balance between capillarity, elasticity

(a)

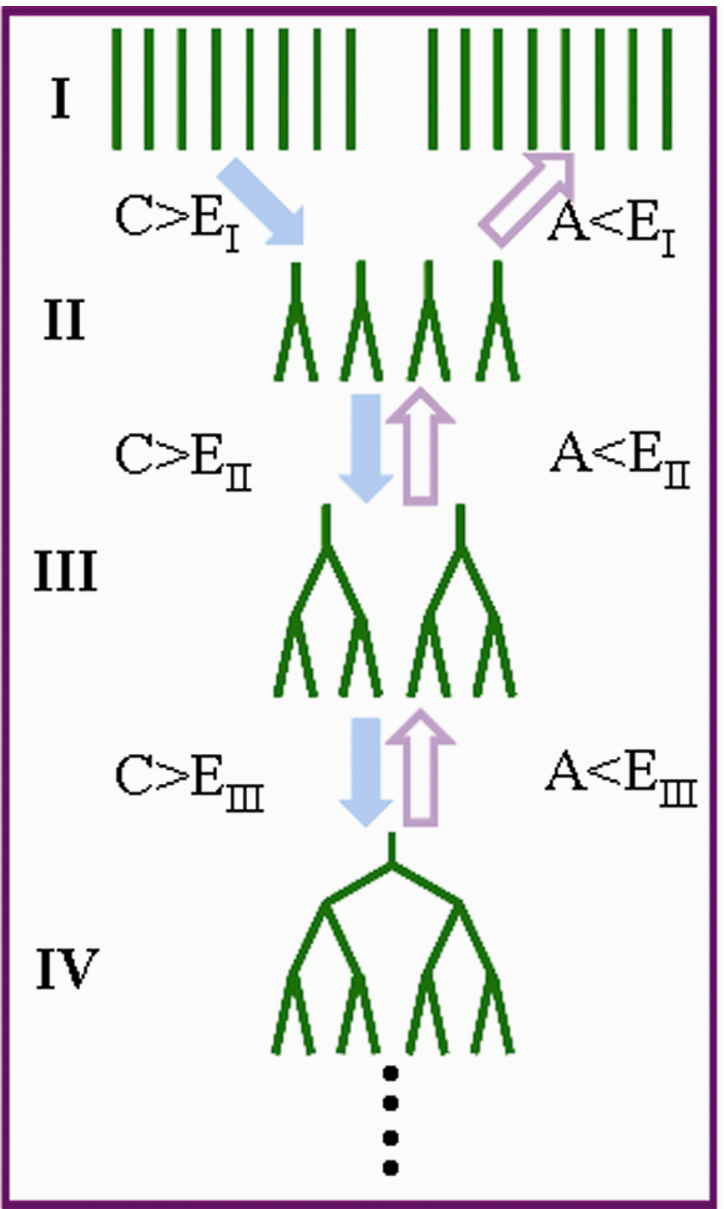

(b)

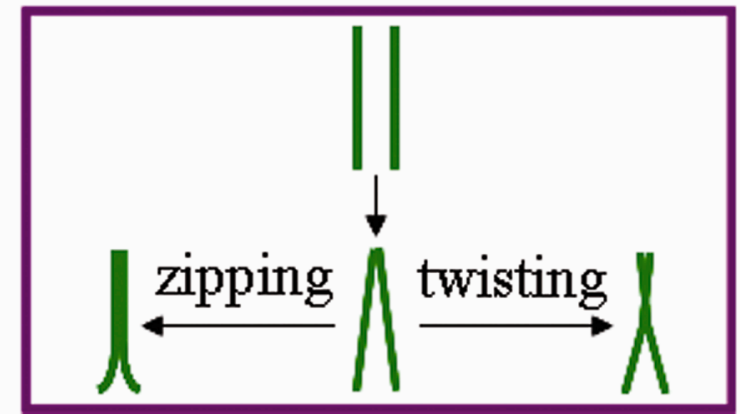

and adhesion.

Figure 6. Schematic diagrams depicting the mechanisms of the assembly process. (a) The possible routes of the assembly and disassembly processes that determine the final pattern. $\mathrm{C}$ and $\mathrm{A}$ indicate the capillary force and the adhesion force, respectively. $\mathrm{E}$ is the elastic force and the subscripts I, II, III, and IV indicate the hierarchy of the assembly. Note that while the interplay between the elastic and capillary force determines the maximum size the assembly can reach while wet, the adhesion force determines stability of the formed clusters and the size and shape of the final product. (b) The different shapes of the assembled clusters. The nanopillars first attached at the tips and depending on the adhesion force between the pillars can either undergo a slippage and chiral rearrangement leading to twisted clusters for low 
adhesion, or zip down the nanopillars for high adhesion values.

The chirality and shape of the assembly are also strikingly dependent on the delicate balance between adhesion, elasticity and capillarity. Pillars initially contact each other at their tips, and chirality arises when the contact point slips and the posts twist around each other (Figure 6b). We have shown that large pillar stiffness can prevent chirality (Fig. 2c, Fig. 4c), most likely by preventing the posts from twisting, ${ }^{32}$ while assemblies are chiral only when the pillars have an intermediate bending modulus (Figure 4b) or diameter (Figure 2b). Similarly, adhesion plays an important role in determining assembly shape and chirality: significantly increasing surface adhesion but not stiffness produces achiral structures in which pillars "zip" together along their length rather than twist (Figure 5 and Figure 6b). This result suggests that, first, chirality can only arise if the adhesion force is low enough to allow the initial slip, and, second, posts can assemble into an alternate shape (e.g. "zipped" structures) that has a large fraction of connected surface area but remains achiral if the adhesion is high enough both to prevent slippage and to stabilize extra bending.

Our results further suggest that the effects of the various parameters on size may themselves be mediated by shape and/or chirality of the forming clusters. Most notably, increasing surface adhesion leads not to larger assemblies that can be sustained by the high adhesion but instead to progressively smaller assemblies (Figure 5). The zippered shape of the four-pillar assembly observed at high adhesion (Figure 5c) may effectively behave as a single pillar with a larger diameter and a shorter height, with less flexibility than if the posts were connected only at their 
tips, possibly analogous to "collaborative stiffening" described for wet hair bundles. ${ }^{40}$ The specific differences between zipped and twisted structures that might make the former less likely to form larger assemblies are unknown, but include differences in effective height or diameter, total contact or exposed area, symmetry, or a more complex liquid-solid contact line, any of which would change either the propensity for capillary-induced bending and stability.

For a more quantitative understanding, we have compared our experimental results for the changes in diameter, modulus, adhesion and wetting of the pillars with theoretical predictions based on the literature ${ }^{25,27}$ using Eq. (1) for various conditions (Figure 7). The experimentally measured average assembly size is consistent with a linear scaling associated with varying modulus and diameter although of the prefactor of 952 is three-fold higher than the value of 258 reported by Chandra et al. ${ }^{25}$ Note that the previous work assumed that the final equilibrium state and the average assembly size is determined by energy minimization that accounts only for elastic bending energy and capillarity energy ${ }^{25}$ However, as we have shown (Fig. 5, 7b), plasma treatment and surface chemistry data points strongly to the importance of adhesion, which has not been considered earlier, and these changes can result in a significant deviation from the expected assembly size based only on elastocapillarity effects.

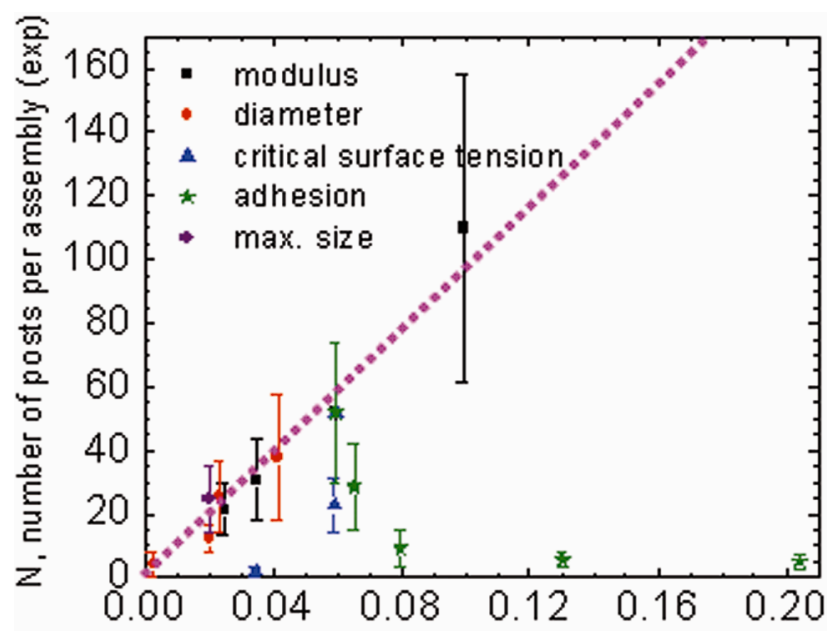

$N$, estimation from $E q$. (1) based on literature
Figure 7. The comparison of the experimental data for the number of posts per assembly vs. theoretical estimation based on eq. (1) for the modulus, diameter, critical surface tension and adhesion change, respectively. The data point for the maximum size is from the data before 
complete drying shown in Fig. 2 (d). The slope of the dotted line is 952.

More generally, the existing static models fail to reflect the dynamic aspect of the process associated with the hierarchical nature of assembly and disassembly (Figure 6a). If the collective forces driving slippage or chiral/achiral increases in surface contact are strong enough relative to those driving higher-level association, the strong local interactions due to adhesion may effectively modify parameters such as diameter, height, exposed area, or symmetry as discussed. For example, the self-assembly of pillars ${ }^{32}$ can lead to the collaborative stiffening and adhesion of the structures ${ }^{40}$ and thus a complex evolution of the shape of self-assembled clusters, so that a global minimum energy state ${ }^{41}$ may never be reached. Ultimately, any comprehensive model for capillary-induced assembly must account for the dynamical interplay between capillarity, elasticity and adhesion, on different length scales.

Finally, our study shows that manipulating the properties of the building blocks of selfassembly, including geometry, anisotropy, stiffness and surface chemistry of assembling nanofibers, provides a simple way of fabricating complex hierarchical nanostructures, which can be tailored to have desired tunable functions for applications such as trapping and releasing of nano- and microparticles, controlling wetting properties by changing the surface morphology, ${ }^{42}$ adhesives based on mechanical interlocking, ${ }^{32}$ and controlling optical properties by structural effects, ${ }^{25,43}$ to name a few.

\section{Conclusions}

Using a model self-assembly system with easily tunable parameters, we have demonstrated that varying the mechanical and/or surface properties of the nanopillars provides us with simple 
routes for fabricating complex structures with control of size, chirality and anisotropy by capillary-induced self-organization. Our results also showed that it would be necessary to consider adhesion as well as the kinetics of assembly for a more comprehensive understanding and prediction of assembly patterns. By varying the geometry, stiffness and chemistry of the assembling nanopillars, we observe that chirality can be generated within a small window for each parameter, even though the systems do not involve either chiral building blocks or a chiral environment. Moreover, the use of anisotropic building blocks expands our toolbox for controlling the size, geometry and chirality of the assembled clusters. This study provides a broader perspective of how a variety of features contribute collectively to capillary-induced selfassembly and demonstrates many options for fine tuning the assembly to generate diverse structures.

\section{Experimental Methods}

Fabrication of fibrous surfaces with controlled parameters: Four silicon masters were used in this study. The master \#1 had arrays of pillars with a diameter of $250 \mathrm{~nm}$, a height of $8 \mu \mathrm{m}$ and a pitch of $2 \mu \mathrm{m}$. The pillars surfaces showed a pronounced scalloping at the side walls due to the fabrication process. This master was used in most parts of this study. Replicas with different moduli and geometries were fabricated from this master using soft lithography-based methods (Figure 1). The master \#2 had arrays of pillars with a diameter of $1700 \mathrm{~nm}$, a height of $10 \mu \mathrm{m}$ and a pitch of $3.5 \mu \mathrm{m}$. The master \#3 had the same lateral dimensions as \#1 and a height of 5 $\mu \mathrm{m}$. The master \#4 has the same feature sizes as the master \#1, but it had no scalloping at the top half of the pillars. For all samples, the polymer replicas of the fibrous surface were fabricated using the method described in reference. ${ }^{33}$ The patterned pillar array covered an area of $10 \times 10$ $\mathrm{mm}^{2}$. With the exception for the modulus study, all posts were made from commercially 
available UV-curable epoxy (UVO-114 from Epoxy Technology, Billerica, MA). To vary the modulus of the replica, different concentrations of either a softener (Dow DER 732) or silicon dioxide nanoparticles were added to the epoxy resin. The details of tuning the modulus of the material by mixing two kinds of epoxies were described in our previous report. ${ }^{33}$ The silica nanoparticles were added to increase the modulus of the posts beyond that of the harder epoxy. In this case, a small amount (2 wt $\%$ or $4 \mathrm{wt} \%)$ of hexamethyldisilazane-treated silica nanoparticles (available from Gelest, Inc.) was mixed with epoxy resin by sonication with a Branson digital sonifier, and the mixture was put in a vacuum to remove bubbles before putting it in a negative mold and curing with UV. The flexure modulus of the composite materials were measured using the 4 point bending test with an Instron 5566 universal materials testing machine by making bulk specimens. All modulus values used in this study are from the measurement of bulk specimens.

To vary the diameter of the post, new negative molds with adjusted sizes of wells were made by the following procedures. To increase the diameter of the replica without changing other parameters, metal films of controlled thickness were deposited on the initial epoxy replica by using the AJA sputtering system of the Harvard Center for Nanoscale System and then the coated replica was used to make negative molds with increased well diameters. From the new molds, epoxy replicas with increased pillar diameters were made. For reduction of the diameter of the pillars, plasma etching by Femto plasma cleaner (Diener electronic $\mathrm{GmbH}$ ) was used to thin down the epoxy replica, followed by formation of negative molds with reduced well diameters and fabrication of replicas with reduced pillar diameters from the new negative molds.

To control the surface wetting properties, surface functionalization using silanes with different critical surface tension values were used. After fabricating epoxy fibrous surfaces, the 
samples were treated with plasma for 10 seconds using Femto plasma cleaner to activate the $\mathrm{OH}$ groups of epoxy. A couple of drops of silane were then put in a glass vial with the samples in a desiccator, which was connected to vacuum. For a partially wetting surface, Heptadecafluoro1,1,2,2-Tetrahydrodecyl Tricholorosilane (available from Gelest, Inc.) was used, and for a wetting surface, p-Tolyltrichlorosilane (available from Sigma Aldrich, Co.) was used. After overnight deposition, the sample was blow-dried with nitrogen. For fabricating fibrous surfaces with anisotropic properties, the negative elastomer mold was pulled along various lattice directions using clamps, and the epoxy resin was put and cured while keeping the mold in the deformed state. ${ }^{33}$

Characterization of assembly: Self-assembly was induced by placing a small volume (10-20 $\mu \mathrm{L})$ of 200 proof absolute anhydrous ethanol (Pharmco-AAPER) on a sample to cover the entire area and letting the solvent evaporate under ambient conditions. As the solvent evaporated, the posts assembled and retained a certain assembly pattern after the system was completely dried (Figure 1a). Then, the sample was coated with Pt/Pd for $60 \mathrm{sec}$ at $40 \mathrm{~mA}$ current with Cressington 208 HR sputter coater for imaging and characterized by JEOL JSM-639 OLV scanning electron microscope and Zeiss Ultra 55 scanning electron microscope.

ACKNOWLEDGMENT This work was partially supported by the Materials Research Science and Engineering Center under NSF award no. DMR-0820484. We acknowledge the use of the facilities at the Harvard Center for Nanoscale Systems supported by NSF award no. ECS0335765. We thank Dr. Alison Grinthal for her help to prepare the manuscript. SHK thanks Drs. Ning Wu and Alison Grinthal for helpful discussions.

Supporting Information Available: Details of analysis of the assembly size for the anisotropic nanopillar arrays, effects of changing the wetting properties of the nanopillar arrays, and effects 
of corrugation of the walls of the pillars. This material is available free of charge via the Internet at http://pubs.acs.org.

\section{REFERENCES}

1. Lehn, J. M. Perspectives in Supramolecular Chemistry - from Molecular Recognition Towards Molecular Information-Processing and Self-Organization. Angew. Chem. Int. Ed. 1990, 29, 1304-1319.

2. Whitesides, G. M.; Mathias, J. P.; Seto, C. T. Molecular Self-Assembly and Nanochemistry - a Chemical Strategy for the Synthesis of Nanostructures. Science 1991, 254, 1312-1319.

3. Whitesides, G. M.; Grzybowski, B. Self-Assembly at All Scales. Science 2002, 295, 2418-2421.

4. Israelachvili, J. N.; Mitchell, D. J.; Ninham, B. W. Theory of Self-Assembly of Hydrocarbon Amphiphiles into Micelles and Bilayers. J. Chem. Soc., Faraday Trans. 2 1976, 72 , 1525-1568.

5. Boncheva, M.; Bruzewicz, D. A.; Whitesides, G. M. Millimeter-Scale Self-Assembly and Its Applications. Pure Appl. Chem. 2003, 75, 621-630.

6. Stauth, S. A.; Parviz, B. A. Self-Assembled Single-Crystal Silicon Circuits on Plastic. Proc. Natl. Acad. Sci. U. S. A. 2006, 103, 13922-13927.

7. Grzybowski, B. A.; Stone, H. A.; Whitesides, G. M. Dynamic Self-Assembly of Magnetized, Millimetre-Sized Objects Rotating at a Liquid-Air Interface. Nature 2000, 405, 1033-1036.

8. Tien, J.; Terfort, A.; Whitesides, G. M. Microfabrication through Electrostatic SelfAssembly. Langmuir 1997, 13, 5349-5355.

9. Adams, M.; Dogic, Z.; Keller, S. L.; Fraden, S. Entropically Driven Microphase Transitions in Mixtures of Colloidal Rods and Spheres. Nature 1998, 393, 349-352.

10. Bowden, N.; Terfort, A.; Carbeck, J.; Whitesides, G. M. Self-Assembly of Mesoscale Objects into Ordered Two-Dimensional Arrays. Science 1997, 276, 233-235.

11. Jacobs, H. O.; Tao, A. R.; Schwartz, A.; Gracias, D. H.; Whitesides, G. M. Fabrication of a Cylindrical Display by Patterned Assembly. Science 2002, 296, 323-325.

12. Rothemund, P. W. K. Using Lateral Capillary Forces to Compute by Self-Assembly. Proc. Natl. Acad. Sci. U. S. A. 2000, 97, 984-989.

13. Smela, E.; Inganas, O.; Lundstrom, I. Controlled Folding of Micrometer-Size Structures. Science 1995, 268, 1735-1738.

14. Srinivasan, U.; Liepmann, D.; Howe, R. T. Microstructure to Substrate Self-Assembly Using Capillary Forces. J. MEMS 2001, 10, 17-24.

15. Syms, R. R. A. Surface Tension Powered Self-Assembly of 3-D Micro-Optomechanical Structures. J. MEMS 1999, 8, 448-455.

16. Bico, J.; Roman, B.; Moulin, L.; Boudaoud, A. Elastocapillary Coalescence in Wet Hair. Nature 2004, 432, 690-690. 
17. Geim, A. K.; Dubonos, S. V.; Grigorieva, I. V.; Novoselov, K. S.; Zhukov, A. A.; Shapoval, S. Y. Microfabricated Adhesive Mimicking Gecko Foot-Hair. Nature Mater. 2003, 2 , 461-463.

18. Chen, R.; Lu, M. C.; Srinivasan, V.; Wang, Z.; Cho, H. H.; Majumdar, A. Nanowires for Enhanced Boiling Heat Transfer. Nano Lett. 2009, 9, 548-553.

19. Lau, K. K. S.; Bico, J.; Teo, K. B. K.; Chhowalla, M.; Amaratunga, G. A. J.; Milne, W. I.; McKinley, G. H.; Gleason, K. K. Superhydrophobic Carbon Nanotube Forests. Nano Lett. 2003, 3, 1701-1705.

20. De Volder, M.; Tawfick, S. H.; Park, S. J.; Copic, D.; Zhao, Z.; Lu, W.; Hart, A. J. Diverse 3d Microarchitectures Made by Capillary Forming of Carbon Nanotubes. Adv. Mater. 2010, DOI: 10.1002/adma.201001893.

21. Duan, H.; Berggren, K. K. Directed Self-Assembly at the $10 \mathrm{Nm}$ Scale by Using Capillary Force-Induced Nanocohesion. Nano Lett. 2010, 10, 3710-3716.

22. Eisner, T.; Aneshansley, D. J. Defense by Foot Adhesion in a Beetle (Hemisphaerota Cyanea). Proc. Natl. Acad. Sci. U. S. A. 2000, 97, 6568-6573.

23. Kim, H. Y.; Mahadevan, L. Capillary Rise between Elastic Sheets. J. Fluid Mech. 2006, 548, 141-150.

24. Py, C.; Bastien, R.; Bico, J.; Roman, B.; Boudaoud, A. 3d Aggregation of Wet Fibers. Europhys. Lett. 2007, 77, 44005.

25. Chandra, D.; Yang, S.; Soshinsky, A. A.; Gambogi, R. J. Biomimetic Ultrathin Whitening by Capillary-Force-Induced Random Clustering of Hydrogel Micropillar Arrays. ACS Appl. Mater. Interfaces 2009, 1, 1698-1704.

26. Chandra, D.; Yang, S. Stability of High-Aspect-Ratio Micropillar Arrays against Adhesive and Capillary Forces. Acc. Chem. Res. 2010, 43, 1080-1091.

27. Zhao, Y. P.; Fan, J. G. Clusters of Bundled Nanorods in Nanocarpet Effect. Appl. Phys. Lett. 2006, 88, 103123.

28. Lichtenegger, H.; Muller, M.; Paris, O.; Riekel, C.; Fratzl, P. Imaging of the Helical Arrangement of Cellulose Fibrils in Wood by Synchrotron X-Ray Microdiffraction. J. Appl. Crystallogr. 1999, 32, 1127-1133.

29. Wagermaier, W.; Gupta, H. S.; Gourrier, A.; Burghammer, M.; Roschger, P.; Fratzl, P. Spiral Twisting of Fiber Orientation inside Bone Lamellae. Biointerphases 2006, 1, 1-5.

30. Jewell, S. A.; Vukusic, P.; Roberts, N. W. Circularly Polarized Colour Reflection from Helicoidal Structures in the Beetle Plusiotis Boucardi. New J. Phys. 2007, 9, 99.

31. Vukusic, P.; Sambles, J. R.; Lawrence, C. R.; Wootton, R. J. Quantified Interference and Diffraction in Single Morpho Butterfly Scales. Proc. R. Soc. B 1999, 266, 1403-1411.

32. Pokroy, B.; Kang, S. H.; Mahadevan, L.; Aizenberg, J. Self-Organization of a Mesoscale Bristle into Ordered, Hierarchical Helical Assemblies. Science 2009, 323, 237-240.

33. Pokroy, B.; Epstein, A. K.; Persson-Gulda, M. C. M.; Aizenberg, J. Fabrication of Bioinspired Actuated Nanostructures with Arbitrary Geometry and Stiffness. Adv. Mater. 2009, $21,463-469$.

34. Webb, J. F. Neuromast Morphology and Lateral Line Trunk Canal Ontogeny in 2 Species of Cichlids - an Sem Study. J. Morphol. 1989, 202, 53-68.

35. Autumn, K.; Liang, Y. A.; Hsieh, S. T.; Zesch, W.; Chan, W. P.; Kenny, T. W.; Fearing, R.; Full, R. J. Adhesive Force of a Single Gecko Foot-Hair. Nature 2000, 405, 681-685.

36. Gao, X.; Jiang, L. Biophysics: Water-Repellent Legs of Water Striders. Nature 2004, 432,36 . 
37. Boudaoud, A.; Bico, J.; Roman, B. Elastocapillary Coalescence: Aggregation and Fragmentation with a Maximal Size. Phys. Rev. E 2007, 76, 060102.

38. Liston, E. M.; Martinu, L.; Wertheimer, M. R. Plasma Surface Modification of Polymers for Improved Adhesion - a Critical-Review. J. Adhes. Sci. Technol. 1993, 7, 1091-1127.

39. Journet, C.; Moulinet, S.; Ybert, C.; Purcell, S. T.; Bocquet, L. Contact Angle Measurements on Superhydrophobic Carbon Nanotube Forests: Effect of Fluid Pressure. Europhys. Lett. 2005, 71, 104-109.

40. Chiodi, F.; Roman, B.; Bico, J. Piercing an Interface with a Brush: Collaborative Stiffening. Europhys. Lett. 2010, 90, 44006.

41. Bernardino, N.; Dietrich, S. Comment on Biomimetic Ultrathin Whitening by CapillaryForce-Induced Random Clustering of Hydrogel Micropillar Arrays. ACS Appl. Mater. Interfaces 2010, 2, 603-604.

42. Lee, J. S.; Ryu, J.; Park, C. B. Bio-Inspired Fabrication of Superhydrophobic Surfaces through Peptide Self-Assembly. Soft Mater. 2009, 5, 2717-2720.

43. Vukusic, P.; Hallam, B.; Noyes, J. Brilliant Whiteness in Ultrathin Beetle Scales. Science 2007, 315, 348-348.

\section{Graphical Table of Contents}
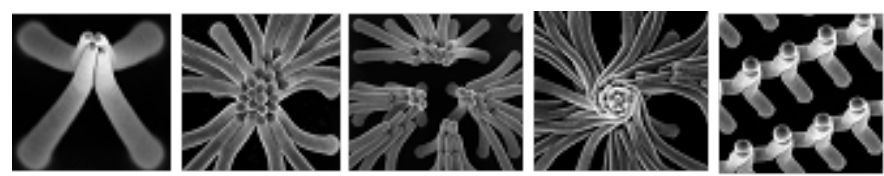